\title{
Synergistic effects of oxyresveratrol in conjunction with antibiotics against methicillin-resistant Staphylococcus aureus
}

\author{
DAE-KI JOUNG ${ }^{1 *}$, SUNG-HOON CHOI ${ }^{*}$, OK-HWA KANG ${ }^{1}$, SUNG-BAE KIM $^{2}$, SU-HYUN MUN ${ }^{2}$, \\ YUN-SOO SEO ${ }^{1}$, DA-HYE KANG ${ }^{1}$, RYONG GONG ${ }^{2}$, DONG-WON SHIN ${ }^{3}$, \\ YOUN-CHUL KIM ${ }^{4}$ and DONG-YEUL KWON ${ }^{1}$
}

\author{
${ }^{1}$ College of Pharmacy and Wonkwang-Oriental Medicines Research Institute, Institute of Biotechnology, \\ Wonkwang University, Iksan, Jeonbuk 570-749; ${ }^{2}$ BK21 Plus Team, Professional Graduate School of Oriental Medicine, \\ Wonkwang University, Iksan, Jeonbuk 570-749; ${ }^{3}$ Department of Oriental Medicine Resources, \\ Sunchon National University, Sunchon, Jeonnam 540-742; ${ }^{4}$ Standardized Material Bank for New Botanical Drugs, \\ College of Pharmacy, Wonkwang University, Iksan 570-749, Republic of Korea
}

Received December 2, 2013; Accepted July 9, 2014

DOI: $10.3892 / \mathrm{mmr} .2015 .3345$

\begin{abstract}
Methicillin-resistant Staphylococcus aureus (MRSA) infection is a serious clinical problem worldwide. The aim of the present study was to examine the antimicrobial activity of oxyresveratrol (ORV) against MRSA. The antimicrobial activity of ORV was evaluated against three strains of MRSA and one methicillin-susceptible S. aureus (MSSA) strain using a minimal inhibitory concentration (MIC) assay, MTT colorimetric assay, checkerboard dilution test and time-kill assay. The MIC of ORV for all strains was moderate at $125 \mu \mathrm{g} / \mathrm{ml}$. Of note, the antimicrobial activity and fractional inhibitory concentration index values of ORV were markedly increased in the presence of a non-growth inhibitory dose of certain antibiotics. Time-kill curves revealed that a combination of ORV with ciprofloxacin or with gentamicin reduced bacterial counts to below the lowest detectable limit after $24 \mathrm{~h}$. These effective combinations may be used as potential antimicrobial regimens for use in the management of MRSA.
\end{abstract}

\section{Introduction}

Staphylococcus aureus is a bacterium that grows in the human nose and skin and is a major pathogen that causes skin and soft-tissue infections, which have previously been

Correspondence to: Dr Dong-Yeul Kwon, College of Pharmacy and Wonkwang-Oriental Medicines Research Institute, Institute of Biotechnology, Wonkwang University, 344-2 Shinyong-dong, Iksan, Jeonbuk 570-749, Republic of Korea

E-mail: sssimi@wku.ac.kr

${ }^{*}$ Contributed equally

Key words: methicillin-resistant Staphylococcus aureus, oxyresveratrol, synergism, antimicrobial treated with the antibiotic methicillin. Since its detection in 1961, methicillin-resistant $S$. aureus (MRSA) has become the most problematic Gram-positive bacterium in the public health arena (1). This pathogen is associated with a variety of infectious diseases (2) and has an average mortality rate of $36-50 \%$ (3). With increasing resistance to various antibiotics, combination therapy is a potential alternative. It may prove particularly useful in developing countries where availability of antibiotics is limited, as it allows for a reduction in the dose of the antibiotic required (4-6). Furthermore, MRSA bacteria are not only resistant to $\beta$-lactam antibiotics but also to fluoroquinolones and other families of antibiotics (4).

Oxyresveratrol (ORV) is an antioxidant (7), anthelmintic (8) tyrosinase inhibitor (9) and a cyclooxygenase inhibitor $(10,11)$. Various studies have indicated that oxyresveratrol (Fig. 1) inhibits apoptotic cell death in transient cerebral ischemia (12), is hepatoprotective (13) and is a potent free radical scavenger (7). Oxyresveratrol has been demonstrated to have an inhibitory effect on the herpes simplex and varicella zoster virus $(14,15)$. In addition, the compound has been revealed to have skin-whitening (16) and neuroprotective effects $(6,7,13,14)$. However, the antimicrobial capacity of ORV against Staphylococcus aureus remains unknown. Therefore, the antibacterial activity of ORV alone and of ORV in conjunction with commonly-used antibiotics was investigated in the present study.

\section{Materials and methods}

Materials and chemicals. Ampicillin (AM), oxacillin (OX), gentamicin (GT), vancomycin (VC), norfloxacin (NR) and ciprofloxacin (CP) (all supplied by Sigma-Aldrich, St. Louis, MO, USA) were used. Oxyresveratrol ( $>96.32 \%)$ was deposited at the Standardized Material Bank for New Botanical Drugs (No NNMBP000018) at Wonkwang University (Iksan, Republic of Korea). The twigs of Morus alba were purchased from the herbal medicine co-operative association of Jeonbuk Province, Korea, in October 2000. A voucher specimen 
Table I. Interpreted FICI response against MRSA and MSSA strains.

A, Response for ORV and AM

\begin{tabular}{|c|c|c|c|c|c|}
\hline \multirow[b]{2}{*}{ Strain } & \multicolumn{2}{|c|}{$\mathrm{ORV}^{\mathrm{a}}$} & \multicolumn{2}{|c|}{$\mathrm{AM}^{\mathrm{a}}$} & \multirow[b]{2}{*}{ FICI } \\
\hline & Alone & With AM & Alone & With ORV & \\
\hline ATCC 33591 & 125 & 62.5 & 1,000 & 250 & 0.75 \\
\hline ATCC 25923 & 125 & 15.6 & 31.25 & 1.95 & 0.1875 \\
\hline DPS-1 & 125 & 31.25 & 1,000 & 500 & 0.75 \\
\hline DPS-2 & 125 & 62.5 & 1,000 & 500 & 1.00 \\
\hline
\end{tabular}

B, Response for ORV and OX

\begin{tabular}{|c|c|c|c|c|c|}
\hline \multirow[b]{2}{*}{ Strain } & \multicolumn{2}{|c|}{$\mathrm{ORV}^{\mathrm{a}}$} & \multicolumn{2}{|c|}{$O X^{a}$} & \multirow[b]{2}{*}{ FICI } \\
\hline & Alone & With OX & Alone & With ORV & \\
\hline ATCC 33591 & 125 & 62.5 & 500 & 250 & 1 \\
\hline ATCC 25923 & 125 & 15.6 & 125 & 7.81 & 0.1875 \\
\hline DPS-1 & 125 & 62.5 & 1,000 & 62.5 & 0.5625 \\
\hline DPS-2 & 125 & 62.5 & 1,000 & 250 & 0.75 \\
\hline
\end{tabular}

C, Response for ORV and GT

\begin{tabular}{lcccrr}
\hline & \multicolumn{3}{c}{ ORV $^{\mathrm{a}}$} & & \multicolumn{2}{c}{ GT $^{\mathrm{a}}$} \\
\cline { 2 - 3 } Strain & Alone & With GT & & Alone & With ORV \\
\hline ATCC 33591 & 125 & 62.5 & 31.25 & 3.9 & 0.625 \\
ATCC 25923 & 125 & 31.25 & 62.5 & 0.375 \\
DPS-1 & 125 & 62.5 & 250 & 15.6 & 0.5625 \\
DPS-2 & 125 & 62.5 & 125 & 31.25 \\
\hline
\end{tabular}

$\mathrm{D}$, Response for ORV and VC

\begin{tabular}{lccccc}
\hline & \multicolumn{2}{c}{ ORV $^{\mathrm{N}}$} & \multicolumn{2}{c}{ VC $^{\mathrm{a}}$} \\
\cline { 2 - 3 } Strain & Alone & With VC & Alone & With ORV \\
\hline ATCC 33591 & 125 & 31.25 & 1.95 & 0.24 & 0.375 \\
ATCC 25923 & 125 & 62.5 & 3.9 & 1.95 & 0.98 \\
DPS-1 & 125 & 62.5 & 3.9 & 0.98 & 0.5 \\
DPS-2 & 125 & 31.25 & & 1
\end{tabular}

E, Response for ORV and CP

\begin{tabular}{lcccrr}
\hline & \multicolumn{2}{c}{ ORV $^{\mathrm{a}}$} & \multicolumn{2}{c}{ CP $^{\mathrm{a}}$} \\
\cline { 2 - 5 } Strain & Alone & With CP & Alone & With ORV \\
\hline ATCC 33591 & 125 & 31.25 & 500 & 62.5 \\
ATCC 25923 & 125 & 15.6 & 31.25 & 3.9 & 0.375 \\
DPS-1 & 125 & 15.6 & 125 & 31.25 & 0.25 \\
DPS-2 & 125 & 31.25 & 125 & 31.25 \\
\hline
\end{tabular}


Table I. Continued.

F, Response for ORV and NR

\begin{tabular}{llccrr}
\hline & \multicolumn{2}{c}{ ORV $^{\mathrm{a}}$} & \multicolumn{3}{c}{ NR $^{\mathrm{a}}$} \\
\cline { 2 - 3 } Strain & Alone & With NR & Alone & With ORV \\
\hline ATCC 33591 & 125 & 62.5 & 250 & 62.5 & 0.75 \\
ATCC 25923 & 125 & 62.5 & 15.6 & 3.9 & 0.75 \\
DPS-1 & 125 & 62.5 & 31.25 & 15.6 & 1 \\
DPS-2 & 125 & 62.5 & 31.25 & 7.8 & 0.75 \\
\hline
\end{tabular}

${ }^{a}$ Minimun inhibitory concentration $(\mu \mathrm{g} / \mathrm{ml})$. FICI, fractional inhibitory concentration index; MRSA, methicillin-resistant $S$. aureus; MSSA, methicillin-susceptible S. aureus. ORV, oxyresveratrol; AM, ampicillin; OX, oxacillin; GT, gentamicin; VC, vancomycin; CP, ciprofloxacin; NR, norfloxacin.

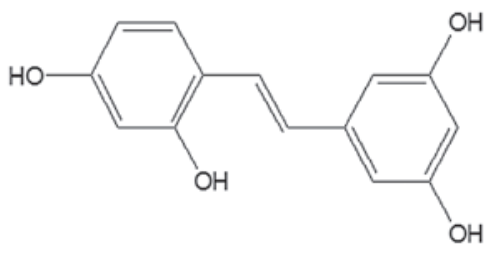

Figure 1. Structure of oxyresveratrol.

(no. WP 217) was deposited at the Herbarium of the College of Pharmacy, Wonkwang University (Korea). Dried twigs of M. alba (2 kg) were extracted with EtOH (2 1) for 20 days at room temperature. Dried residue of the EtOH extract (101 g) was dissolved in $40 \%$ aqueous $\mathrm{MeOH}(1 \mathrm{~L})$ and partitioned with n-hexane (800 $\mathrm{ml} \mathrm{x2}), \mathrm{CH}_{2} \mathrm{Cl}_{2}(800 \mathrm{ml} \mathrm{x} 2)$ and EtOAc (800 ml x2), successively. The $\mathrm{CH}_{2} \mathrm{Cl}_{2}$ soluble fraction $(8.53 \mathrm{~g}$ ) was chromatographed on Sephadex LH-20 column $(5 \mathrm{x} 16 \mathrm{~cm})$ using $\mathrm{CH}_{2} \mathrm{Cl}_{2}-\mathrm{MeOH}$ (4:1 - 1:1; each volume, $\left.300 \mathrm{ml}\right)$ to obtain four fractions (Fr. A-D). The EtOAc soluble fraction (4.83 g) was chromatographed on silica gel (250 g) column using $\mathrm{CH}_{2} \mathrm{Cl}_{2}-\mathrm{MeOH}$ (8:1 - 4:1; each volume, $600 \mathrm{ml}$ ) to obtain three fractions (Fr. D-F). Fr. E (2.77 g) was chromatographed on silica gel $(150 \mathrm{~g})$ column (eluent: n-hexane-acetone, 1:1) and further purified by Sephadex LH-20 column $(2.5 \times 20 \mathrm{~cm})$ chromatography (eluent, $\mathrm{CH}_{2} \mathrm{Cl}_{2}-\mathrm{MeOH} 4: 1$ ) to give oxyresveratrol $(1.12 \mathrm{~g}, 0.056 \mathrm{w} / \mathrm{w} \%)$. The structure of oxyresveratrol was identified by analysis of nuclear magnetic resonance and mass spectra.

Bacterial strains and growth conditions. Two clinical MRSA isolates were obtained from two patients at Wonkwang University Hospital (Iksan, South Korea). The other two strains were obtained from the American Type Culture Collection (Manassas, VA, USA), and included S. aureus (33591; methicillin-resistant strain) and S. aureus (25923; methicillin-susceptible strain). Prior to each experiment, all bacteria were stored in $30 \%$ glycerol and frozen at $-70^{\circ} \mathrm{C}$. The bacteria were cultured in Difco ${ }^{\mathrm{TM}}$ Mueller-Hinton broth (MHB) and Mueller-Hinton agar (MHA) (BD Biosciences, Franklin Lakes, NJ, USA) by incubating at $37^{\circ} \mathrm{C}$ for $24 \mathrm{~h}$.
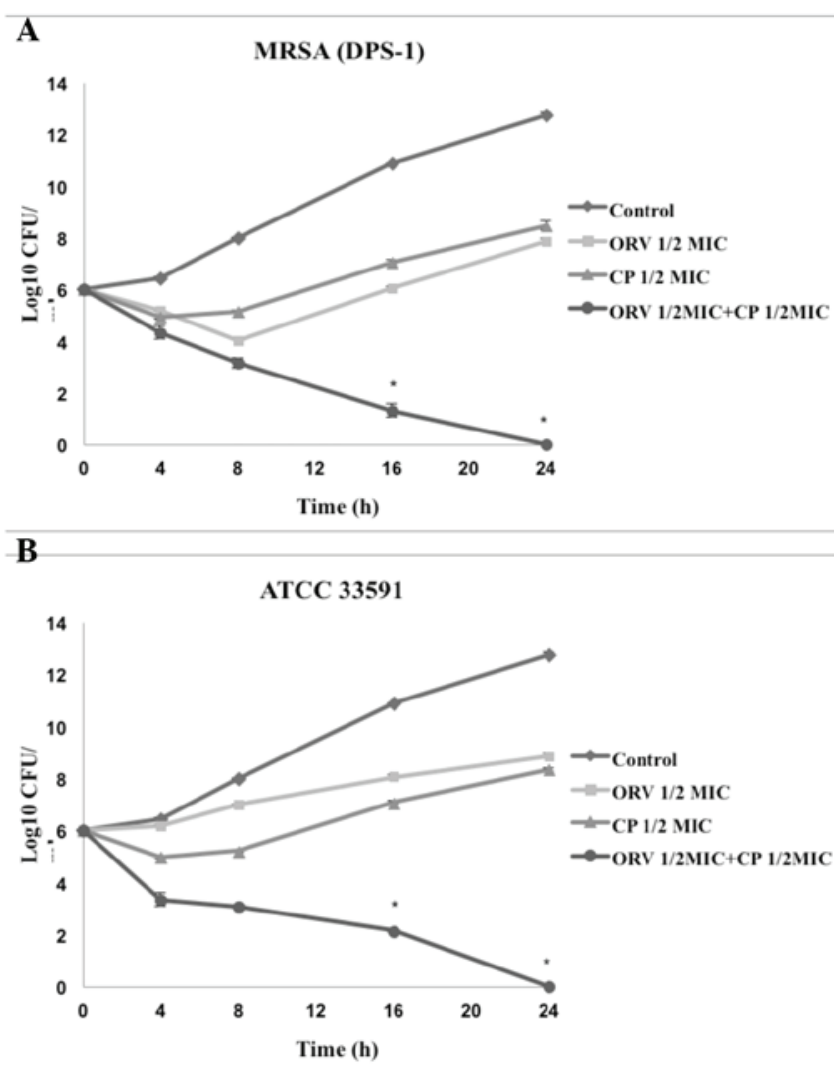

Figure 2. Time-kill curves for combined ORV and CP treatment of the (A) DPS-1 MRSA and (B) standard 33591 MRSA strains. Data represent the average of triple-indipendent experiments. ${ }^{*} \mathrm{P}<0.001$ as compared to $\mathrm{CP}$ alone. ORV, oxyresveratrol; $\mathrm{CP}$, ciproflaxin; MIC, minimal inhibitory concentration; MRSA, methicillin-resistant S. aureus.

Minimum inhibitory concentration (MIC). MICs were determined using the broth microdilution method according to the guidelines of the Clinical and Laboratory Standards Institute (17). Briefly, a preparation of microorganism suspension was prepared by growing the bacteria in broth for $24 \mathrm{~h}$, which were adjusted to a $0.5 \mathrm{McFarland}$ standard turbidity $\left[\sim 1.5 \times 10^{8}\right.$ colony forming units $\left.(\mathrm{CFU}) / \mathrm{ml}\right]$. The final inocu- 

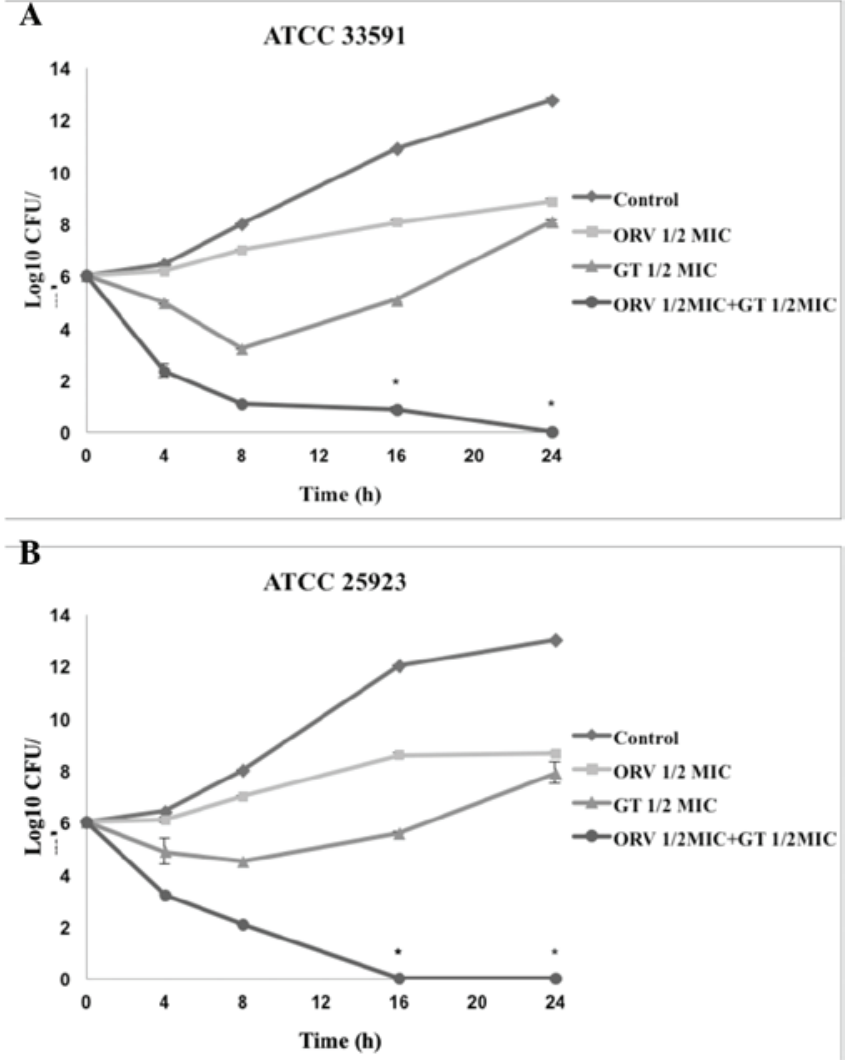

Figure 3. Time-kill curves of ORV and GT against the (A) standard 33591 MSSA and (B) standard 25923 MRSA strains. Data represent the average of triple-indipendent experiments. ${ }^{*} \mathrm{P}<0.001$, as compared with GT alone. ORV, oxyresveratrol; GT, gentamicin; MRSA, methicillin-resistant $S$. aureus; MIC, minimum inhibitory concentration; MSSA, methicillin-susceptible S. aureus.

lums were adjusted to $1.5 \times 10^{6} \mathrm{CFU} / \mathrm{ml}$. The serially diluted extracts were then incubated along with the inoculum at $37^{\circ} \mathrm{C}$ for $18 \mathrm{~h}$. The MIC was defined as the lowest concentration of antibiotics and ORV that prevented visible growth of the bacteria. At the end of the incubation period, well plates were visually examined for turbidity. Cloudiness indicated that bacterial growth had not been inhibited.

Checkerboard dilution test. Synergistic combinations were investigated using the preliminary checkerboard method according to the published standards $(5,18)$. The MIC was defined as the lowest concentration of drug alone or in combination that inhibited visible growth. In vitro interaction was quantified as the fractional inhibitory concentration index (FICI), which was calculated using the following formula: FICI $=($ MIC of drug $\mathrm{A}$ in combination/MIC of drug $\mathrm{A}$ alone) + (MIC of drug B in combination/MIC of drug B alone). FICIs were interpreted as follows: $<0.5$, synergy; 0.5-0.75, partial synergy; 0.76-1.0, additive effect; 1.0-4.0, indifference; and $>4.0$, antagonism. The varying levels of synergy between two given agents were determined (19). All experiments were repeated three times.

MTT colorimetric assay. A colorimetric assay based on MTT for rapid detection of the presence of bacteria was performed as previously described (20-22). Briefly, a stock solution of $5 \mathrm{mg} / \mathrm{ml}$ MTT (Sigma-Aldrich) was prepared in phosphate-buffered saline (Sigma-Aldrich) and stored at $-70^{\circ} \mathrm{C}$. A final concentration of $1 \mathrm{mg} / \mathrm{ml}$ MTT was used in the assay. Following $24-\mathrm{h}$ incubation at $37^{\circ} \mathrm{C}, 20 \mu \mathrm{l}$ yellow MTT was added to a 96-well microtiter plate and incubated for an additional $20 \mathrm{~min}$. Blue color indicated the presence of bacteria.

Time-kill curve assay. A time-kill curve assay was performed according to a previous method (23) in order to investigate the combined effect of time and antimicrobial agent concentration on bacterial growth. For this assay, standard inoculums of $\sim 10^{6} \mathrm{CFU} / \mathrm{ml}$ were used. ORV (0.5 MIC) was used with various combinations of antibiotics ( $0.5 \mathrm{MIC})$. A test plate containing MHB and inoculum was used as the control. Counts of viable strains were conducted at different intervals up to $24 \mathrm{~h}$ at $37^{\circ} \mathrm{C}$. The rate and extent of bacterial death was determined by plotting the viable colony counts $(\mathrm{CFU} / \mathrm{ml})$ against the time cultured in MHA. All experiments were repeated three times.

Statistical analysis. All experiments were performed more than three times. Data from the experiments are presented as the mean \pm standard error of the mean. Statistical analyses were performed using one-way analysis of variance followed by Dunnett's t-test (SPSS software, version 19.0; IBM SPSS, Armonk, NY, USA). $\mathrm{P}<0.001$ was considered to indicate a statistically significant difference.

\section{Results}

MIC determination and synergic effect. Against all strains, the MIC was $125 \mu \mathrm{g} / \mathrm{ml}$ for ORV. VC presented a range of MICs of 1.95-3.9 $\mu \mathrm{g} / \mathrm{ml}$ in the various strains. All strains were resistant to AM, OX, GT, CP and NR, with MIC values ranging from 15.6 to $1,000 \mu \mathrm{g} / \mathrm{ml}$. ORV + antibiotic combinations all exhibited markedly lower MICs than those when the drugs were used alone. The combined use of ORV and VC or CP against the 33591 MRSA strain resulted in a FICI of 0.375 (synergy) (Table I), indicating that an activity-enhancing effect was present. The combined use of ORV and AM or OX against the 33591 MRSA strain resulted in a FICI of 0.75-1, but against the 25923 MSSA strain resulted in a FICI of 0.1875 (synergy). The combined use of ORV and NR against all of the strains resulted in a FICI of 0.75-1 (partial synergy or additive effect). None of the combinations exhibited an antagonistic effect (FICI >4.0). These results demonstrated that the method of combining ORV with antibiotics has potential to be used to suppress MRSA growth.

The controls displayed no reduction in CFU counts, and the use of ORV or antibiotics alone did not induce cell death at $24 \mathrm{~h}$. When used in combination, ORV and antibiotics led to a marked reduction in bacterial counts. In particular, the combination of ORV + GT and ORV + CP completely inhibited growth of $S$. aureus after $24 \mathrm{~h}$. These were the most effective treatments and thus were selected for further analysis.

Time-kill curve assay. Time-kill tests were performed to investigate the synergistic effects of ORV in combination with antibiotics and the effect of length of treatment on cell viability. The control displayed no reduction in CFU counts, 
and the use of ORV or antibiotics alone did not induce cell death at $24 \mathrm{~h}$. When used in combination, ORV and antibiotics caused a marked reduction in bacterial counts. In particular, the combination of ORV + GT and ORV + CP completely inhibited growth of $S$. aureus after 24 h (Figs. 2 and 3).

\section{Discussion}

The most effective method to develop antibiotics that produce minimal toxic effects or side effects is to use natural products. Therefore, there is a requirement for the development of alternative antimicrobial drugs against infectious diseases. Combination therapy is the most commonly recommended empirical treatment for bacterial infections in intensive care units where monotherapy may not be effective against all potential pathogens or for the prevention of antibacterial resistance (24). When combined, certain antibiotics are known to markedly increase bactericidal effects $(5,24,25)$.

To the best of our knowledge, the current study was the first to investigate the potentiation of antibiotics by ORV against MRSA. The MIC assay is considered to be the standard method for determining the susceptibility of various microorganisms to antibacterial agents. The in vitro results of the present study determined the MIC values of ORV and antibiotics against $S$. aureus.

Synergistic or partially synergistic effects of ORV in combination with the antibiotic agents VC, GT and $\mathrm{CP}$ strongly supported this explanation. The time-kill curves and FICI scores confirmed the ability of ORV to synergistically reduce bacterial counts below the lowest detectable limit within $24 \mathrm{~h}$. Therefore, ORV may be a potential antibacterial drug candidate for clinical use against MRSA. The results of the present study are promising and may increase the use of natural products as drugs.

\section{Acknowledgements}

The current study was supported by the Basic Science Research Program through the National Research Foundation of Korea, funded by the Ministry of Education, Science and Technology (grant no. 2012-0004337) and by the Basic Science Research Program through the National Research Foundation of Korea(NRF) funded by the Ministry of Education (grant no. 2013060380).

\section{References}

1. Witte W: Antibiotic resistance in gram-positive bacteria: epidemiological aspects. J Antimicrob Chemoth 44 (Suppl A): 1-9, 1999.

2. Baltch AL, Ritz WJ, Bopp LH, Michelsen PB and Smith RP: Antimicrobial activities of daptomycin, vancomycin, and oxacillin in human monocytes and of daptomycin in combination with gentamicin and/or rifampin in human monocytes and in broth against Staphylococcus aureus. Antimicrob Agents Chemother 51: 1559-1562, 2007.

3. Dancer SJ: The effect of antibiotics on methicillin-resistant Staphylococcus aureus. J Antimicrob Chemother 61: 246-253, 2008.

4. Aqil F, Ahmad I and Owais M: Evaluation of anti-methicillin-resistant Staphylococcus aureus (MRSA) activity and synergy of some bioactive plant extracts. Biotechnol J 1: 1093-1102, 2006.
5. Miranda-Novales G, Leaños-Miranda BE, Vilchis-Pérez M and Solórzano-Santos F: In vitro activity effects of combinations of cephalothin, dicloxacillin, imipenem, vancomycin and amikacin against methicillin-resistant Staphylococcus spp. strains. Ann Clin Microbiol Antimicrob 5: 25, 2006.

6. Kastoris AC, Rafailidis PI, Vouloumanou EK, Gkegkes ID and Falagas ME: Synergy of fosfomycin with other antibiotics for Gram-positive and Gram-negative bacteria. Eur J Clin Pharmacol 66: 359-368, 2010.

7. Lorenz P, Roychowdhury S, Engelmann M, Wolf G and Horn TF: Oxyresveratrol and resveratrol are potent antioxidants and free radical scavengers: effect on nitrosative and oxidative stress derived from microglial cells. Nitric Oxide 9: 64-76, 2003

8. Saowakon N, Tansatit T, Wanichanon C, et al: Fasciola gigantica: anthelmintic effect of the aqueous extract of Artocarpus lakoocha. Exp Parasitol 122: 289-298, 2009.

9. Kim YM, Yun J, Lee CK, et al: Oxyresveratrol and hydroxystilbene compounds. Inhibitory effect on tyrosinase and mechanism of action. J Biol Chem 227: 16340-16344, 2002.

10. Shin NH, Ryu SY, Choi EJ, et al: Oxyresveratrol as the potent inhibitor on dopa oxidase activity of mushroom tyrosinase Biochem Biophys Res Commun 243: 801-803, 1998.

11. Shin NH, Ryu SY, Lee HS, Min KR and Kim YS: Inhibitory effects of hydroxystilbenes on cyclooxygenase from sheep seminal vesicles. Planta Med 64: 283-284, 1998.

12. Ban JY, Jeon SY, Nguyen TT, et al: Neuroprotective effect of oxyresveratrol from smilacis chinae rhizome on amyloid Beta protein (25-35)-induced neurotoxicity in cultured rat cortical neurons. Biol Pharm Bull 29: 2419-2424, 2006.

13. Oh H, Ko EK, Jun JY, et al: Hepatoprotective and free radical scavenging activities of prenylflavonoids, coumarin, and stilbene from Morus alba. Planta Med 68: 932-934, 2002.

14. Chuanasa T, Phromjai J,Lipipun V, et al: Anti-herpes simplex virus (HSV-1) activity of oxyresveratrol derived from Thai medicinal plant: mechanism of action and therapeutic efficacy on cutaneous HSV-1 infection in mice. Antiviral Res 80: 62-70, 2008.

15. Sasivimolphan P, Lipipun V, Likhitwitayawuid K, et al: Inhibitory activity of oxyresveratrol on wild-type and drug-resistant varicella-zoster virus replication in vitro. Antiviral Res 84: 95-97, 2009.

16. Likhitwitayawuid K: Stilbene with tyrosinase inhibitory activity. Curr Sci 94: 44-52, 2008

17. Clinical and Laboratory Standards Institute (CLSI): CLSI documents M7-A5: Methods for dilution antimicrobial susceptibility tests for bacteria that grow aerobically; approved standard. 5th edition. CLSI. Wayne, PA, USA, 2000.

18. Odds FC: Synergy, antagonism, and what the chequerboard puts between them. J Antimicrob Chemother 52: 1, 2003.

19. Mazumdar K, Dutta NK, Kumar KA and Dastidar SG: In vitro and in vivo synergism between tetracycline and the cardiovascular agent oxyfedrine $\mathrm{HCl}$ against common bacterial strains Biol Pharm Bull 28: 713-717, 2005.

20. Scheuber PH, Mossmann H, Beck G and Hammer DK: Direct skin test in highly sensitized guinea pigs for rapid and sensitive determination of staphylococcal enterotoxin B. Appl Environ Microbiol 46: 1351-1356, 1983

21. Abate G, Mshana RN and Miörner H: Evaluation of a colorimetric assay based on 3-(4,5-dimethylthiazol-2-yl)-2,5-diphenyl tetrazolium bromide (MTT) for rapid detection of rifampicin resistance in Mycobacterium tuberculosis. Int J Tuberc Lung Dis 2: 1011-1016, 1998.

22. Shi YJ, Chen J and Xu M: A new method for antimicrobial susceptibility testing of in vitro-cultured bacteria by means of resonance light scattering technique. J Microbiol Biotechnol 18: 118-123, 2008.

23. Nascimento AM, Brandão MG, Oliveira GB, Fortes IC and Chartone-Souza E: Synergistic bactericidal activity of Eremanthus erythropappus oil or beta-bisabolene with ampicillin against Staphylococcus aureus. Antonie Van Leeuwenhoek 92: 95-100, 2007.

24. Drago L, De Vecchi E, Nicola L and Gismondo MR: In vitro evaluation of antibiotics' combinations for empirical therapy of suspected methicillin resistant Staphylococcus aureus severe respiratory infections BMC Infect Dis 7: 111, 2007.

25. Liu IX, Durham DG and Richards RM: Baicalin synergy with beta-lactam antibiotics against methicillin-resistant Staphylococcus aureus and other beta-lactam-resistant strains of S. aureus. J Pharm Pharmacol 52: 361-366, 2000. 